\title{
Colistin Sulfate
}

National Cancer Institute

\section{Source}

National Cancer Institute. Colistin Sulfate. NCI Thesaurus. Code C386.

Cyclic polypeptide antibiotic from Bacillus colistinus. It is composed of Polymyxins E1 and E2 (or Colistins A, B, and C) which act as detergents on cell membranes. Colistin is less toxic than Polymyxin B, but otherwise similar; the methanesulfonate is used orally. (Medical Dictionary Online) 